La Revue

des Droits

de l'Homme

\section{La Revue des droits de l'homme}

Revue du Centre de recherches et d'études sur les droits fondamentaux

Actualités Droits-Libertés | 2019

\title{
La procédure du retrait du bénéfice des conditions matérielles d'accueil des demandeurs d'asile (CE, 31 juillet 2019, Cimade et al., req. $\mathrm{n}^{\circ} 428530$ )
}

\section{Pierre Auriel}

\section{(2) OpenEdition}

\section{Journals}

Electronic version

URL: http://journals.openedition.org/revdh/7571

DOI: 10.4000/revdh.7571

ISSN: 2264-179X

\section{Publisher}

Centre de recherches et d'études sur les droits fondamentaux

\section{Electronic reference}

Pierre Auriel, « La procédure du retrait du bénéfice des conditions matérielles d'accueil des demandeurs d'asile (CE, 31 juillet 2019, Cimade et al., req. $n^{\circ}$ 428530) ", La Revue des droits de l'homme [Online], Actualités Droits-Libertés, Online since 07 November 2019, connection on 11 November 2019. URL : http://journals.openedition.org/revdh/7571; DOI : 10.4000/revdh.7571

This text was automatically generated on 11 November 2019.

Tous droits réservés 


\title{
La procédure du retrait du bénéfice des conditions matérielles d'accueil des demandeurs d'asile (CE, 31 juillet 2019, Cimade et al., req. $\mathrm{n}^{\circ}$ 428530)
}

\author{
Pierre Auriel
}

La réforme de la procédure d'asile issue de la loi du 10 septembre 2018 pour une immigration maitrisée, un droit d'asile effectif et une intégration réussie a alimenté un contentieux portant sur le retrait des conditions matérielles d'accueil ${ }^{1}$, contentieux ayant pour l'heure culminé dans un arrêt du Conseil d'État du 31 juillet $2019^{2}$. Prévues par les articles 17 à 20 de la directive Accueil ${ }^{3}$, les conditions matérielles d'accueil garantissent des conditions de vie décentes aux demandeurs d'asile durant l'examen de leur demande de protection internationale. En France, elles sont gérées par l'office français de l'immigration et de l'intégration et prennent la forme d'une aide financière et de propositions d'hébergement. L'article 20 de la directive expose les comportements des demandeurs d'asile autorisant les États membres à limiter ou à retirer le bénéfice de ces conditions. Les articles L. 744-7 et L.744-8 du Code de l'entrée et du séjour des étrangers et du droit asile modifiés par l'article 9 de la loi du 10 septembre 2018, reprirent l'intégralité des cas envisagés par la directive. Ainsi, l'article L.744-7 impose le retrait lorsque le demandeur d'asile a refusé l'hébergement qui lui était proposé, a quitté le lieu d'hébergement proposé ou a manqué aux obligations imposées par l'administration notamment en ne se rendant pas aux entretiens ou en ne fournissant pas les informations requises. Au titre de l'article L.744-8, l'office français de l'immigration et de l'intégration est autorisé à retirer les conditions matérielles d'accueil lorsque le demandeur d'asile a fourni des informations mensongères à l'administration ou a eu un comportement violent ou manquant aux règles de son lieu d'hébergement. En outre, la loi du 10 septembre 2018 a ajouté un cas qui n'était pas prévu par la directive à l'article L.744-8. Les conditions matérielles d'accueil peuvent 
être retirées lorsque l'individu a " présenté plusieurs demandes d'asile sous des identités différentes ». Cet ajout - dont la conformité à la directive pourrait être discutée - se fonde sur le « principe selon lequel la fraude corrompt tout ${ }^{4}$ ».

2 L'existence de deux articles pour le retrait des conditions matérielles d'accueil traduit une différence de régime. Dans le cadre de l'article L. 744-7, le retrait s'effectue de plein droit et immédiatement ${ }^{5}$. Cette formule assez vague ${ }^{6}$ emporte plus précisément deux conséquences. D'abord, alors que l'article L.744-8 prévoit que le bénéfice des conditions matérielles " peut être retiré » par l'administration, l'article L. 744-7 ne laisse aucune marge de manœuvre à l'administration. Cette dernière ne peut faire dépendre ce retrait de l'éventuelle vulnérabilité du demandeur d'asile. Aucune appréciation au cas par cas n'est possible. Cette situation a une conséquence directe: puisque le retrait est le résultat immédiat et nécessaire du constat d'un comportement d'un demandeur d'asile - absence à un rendez-vous fixé par la préfecture ou départ du logement qui lui a été assigné -, il ne peut pas en demander le rétablissement. En effet, aucune marge de manœuvre n'étant laissée à l'administration, elle ne peut pas prendre en compte une justification de cette absence ou une situation particulière du demandeur d'asile. Elle ne peut donc pas changer de position et une demande de rétablissement est inutile.

3 Ensuite, le retrait dans le cadre de l'article L.744-7, s'effectue sans que le demandeur d'asile ait pu présenter ses observations. Ces deux différences furent précisées par le décret d'application adopté le 28 décembre 2019. En effet, l'article D. 744-37-1 dispose que « la décision de refus ou celle mettant fin aux conditions matérielles d'accueil prise en application de l'article L. 744-7 n'est pas soumise à la mise en œuvre de la procédure prévue à l'article L. 121-1 du code des relations entre le public et l'administration [c'est-à-dire une procédure contradictoire préalable] ». A l'inverse, l'article D. 744-38 prévoit que «la décision mettant fin aux conditions matérielles d'accueil prise en application $\mathrm{du} 1^{\circ}$ de l'article L. 744-8 est écrite, motivée et prise après que le demandeur a été mis en mesure de présenter à l'office français de l'immigration et de l'intégration ses observations écrites dans un délai de quinze jours. Elle prend en compte la vulnérabilité du demandeur ».

4 En outre, le décret d'application a ajouté une autre spécificité au régime de l'article L. 744-7. Tout recours contentieux à l'encontre d'une décision de retrait pris sur ce fondement, doit être précédé d'un recours préalable obligatoire devant le directeur de l'Office, ce dernier devant se prononcer dans un délai de deux mois.

5 Saisi d'un recours formé par des associations de défense de demandeurs d'asile, le Conseil d'État a constaté l'incompatibilité de la procédure de retrait du bénéfice des conditions matérielles d'accueil sur le fondement de l'article L.7 44-7 avec l'article 20 de la directive. Il a estimé notamment qu'« il [résultait] de l'article 20 de la directive que [...] d'une part [le retrait des conditions matérielles d'accueil] ne [pouvait] intervenir qu'après examen de la situation particulière de la personne et être motivé, d'autre part l'intéressé [devait] pouvoir solliciter le rétablissement des conditions matérielles d'accueil lorsque le retrait [avait] été fondé sur l'abandon du lieu de résidence sans information ou autorisation de l'autorité compétente, sur la méconnaissance de l'obligation de se présenter aux autorités ou de se rendre aux rendez-vous qu'elle [fixait] ou sur l'absence de réponse aux demandes d'information». Par conséquent, « en créant des cas de refus et de retrait de plein droit des conditions matérielles d'accueil sans appréciation des circonstances particulières et en excluant, en cas de retrait, toute possibilité de rétablissement de ces conditions, les articles L. 744-7 et L. 
744-8 du code de l'entrée et du séjour des étrangers et du droit d'asile, dans leur rédaction résultant de la loi du 10 septembre 2018, [s'avéraient] incompatibles avec les objectifs de la directive 2013/33/UE du 26 juin $2013^{7} »$. Le Conseil d'État a donc annulé les dispositions réglementaires litigieuses - les articles D. 744-37-1 et D. 744-398. Concernant les dispositions législatives, si l'incompatibilité entre la directive et la loi n'a pas fait pas disparaître cette dernière de l'ordre juridique, elle « fait, en revanche, obstacle à ce que les autorités administratives compétentes adoptent, sur leur fondement, des décisions individuelles mettant fin aux conditions matérielles d'accueil dans des conditions contraires au droit de l'Union" ». Dans le second temps de son raisonnement, le Conseil d'État s'est livré à un exposé des procédures demeurant à la disposition de l'office en attendant la réécriture de la loi par législateur. En particulier, des retraits en raison des motifs prévus par l'article L. 744-7 peuvent être décidés si le demandeur d'asile a été mis en mesure de présenter ses observations au préalable. Le rétablissement du bénéfice des conditions matérielles d'accueil peut être demandé, l'office étant tenu d'apprécier au cas par cas la situation du demandeur d'asile.

$6 \quad$ Le Conseil d'État a ainsi constaté l'incompatibilité de la directive et de la loi tout en permettant la continuation de l'action administrative. À certains égards, la solution du Conseil d'État n'est guère surprenante. Cependant, un point retient l'attention. La décision du 31 juillet 2019 n'est pas la première prise de position du Conseil d'État concernant les dispositions litigieuses. Dans un avis du 15 février 2018, la section de l'intérieur du Conseil d'État avait déclaré ces dispositions conformes aux exigences constitutionnelles et conventionnelles. Elle avait alors uniquement pointé une difficulté concernant la constitutionnalité du mécanisme ${ }^{10}$. Cette divergence est le produit de différences de perspective. Lors de la procédure législative, le mécanisme prévu à l'article L. 744-7 ne fut considéré que comme une partie du dispositif permettant la répartition des demandeurs d'asile dans différentes régions. Les débats se concentrèrent sur la nécessité d'une procédure préalable contradictoire ignorant la difficulté soulevée par l'exigence d'un retrait immédiat et de plein droit. Cette différence de perspective explique en partie que le Conseil d'État ait pu constater la constitutionnalité de ce mécanisme (I) avant de l'écarter en raison de l'incompatibilité entre les exigences du droit de l'Union et un retrait immédiat et de plein droit du bénéfice des conditions matérielles d'accueil (II).

\section{1./ La constitutionnalité de l'absence de procédure préalable contradictoire}

7 L'article L. 744-7 résulte de l'article 9 de la loi du 10 septembre 2018. Cet article a été rédigé et voté afin de mettre en place l'orientation active des demandeurs d'asile. Dans ce cadre, l'article L. 744-7 a été conçu pour être la garantie de cette orientation (A). Dans ce contexte, l'essentiel des discussions se portèrent sur l'absence de procédure contradictoire préalable. En distinguant le retrait des conditions matérielles d'accueil d'une sanction, le Conseil d'État put estimer que cette absence était conforme au principe constitutionnel du respect des droits de la défense (B). 


\section{A. La garantie d'une orientation directive des demandeurs d'asile}

8 Selon l'exposé des motifs de la loi du 10 septembre 2018, l'un des objectifs prioritaires de cette loi était d'endiguer la concentration des demandeurs d'asile en Île-de-France et en Calaisie. À cette fin, le gouvernement introduisit des mécanismes d'orientation active des demandeurs d'asile à l'article 9 de la loi Cet article a lié les conditions matérielles d'accueil au schéma national d'accueil des demandeurs d'asile qui détermine le nombre de demandeurs d'asile que chaque région est tenu d'accueillir. Au moment de l'enregistrement de leur demande, les demandeurs d'asile se voient attribuer un hébergement où ils doivent demeurer s'ils souhaitent bénéficier des conditions matérielles d'accueil. S'ils refusent ou s'ils quittent ce lieu, le bénéfice des conditions est retiré. L'État ne demeure alors tenu que de garantir une aide médicale minimale aux demandeurs d'asile.

9 Lors des débats entourant le projet de loi, ce mécanisme souleva deux critiques. La première concernait l'idée même d'orientation active. En liant une obligation de résidence et le bénéfice des conditions matérielles d'accueil, le projet de loi aurait créé un mécanisme d' " assignation à résidence », voire de " pré-rétention administrative ${ }^{11}$ ». La majorité des demandeurs d'asile n'ayant aucune autre ressource pour survivre que le bénéfice des conditions matérielles d'accueil, ils seraient en réalité privés de leur liberté de circulation.

10 Concernant les modalités des retraits, seule l'absence de procédure contradictoire préalable fit l'objet de débats. Les absences d'appréciation de la situation particulière du demandeur d'asile ou de possibilité pour ce dernier de demander un rétablissement, ne furent pas évoquées. Dans l'exposé des motifs du projet de loi ${ }^{12}$ ou dans l'étude d'impact ${ }^{13}$, le gouvernement a ainsi considéré que la seule nouveauté apportée par le projet de loi dans le régime du retrait du bénéfice des conditions matérielles d'accueil était l'absence de procédure contradictoire préalable dans le cadre de l'article L. 744-7. L'avis du Défenseur des droits ne s'intéressa aussi qu'à cette question. Ainsi, il a estimé que «le fait pour le gouvernement de conférer un effet immédiat à ces décisions de suspension, de retrait ou de refus [placerait] nécessairement les demandeurs d'asile dans une situation de grande difficulté quand bien même une procédure contradictoire [serait] prévue pour les contester a posteriori. Cette nouvelle rédaction [permettrait en effet] aux autorités de priver les demandeurs d'asile de leur hébergement et de l'[Aide aux demandeurs d'asile] avant même que ces personnes n'aient pu présenter leurs observations $s^{14} »$. Les débats devant l'Assemblée ou le Sénat ne portèrent que sur la possibilité d'une extension de l'absence de procédure contradictoire préalable ${ }^{15}$. Les associations de défense des demandeurs d'asile ont aussi ignoré les difficultés soulevées par l'absence d'appréciation de la situation particulière du requérant. Ainsi, dans son analyse de la loi, la Cimade a uniquement relevé que dans le projet de loi, «il [était] prévu, à l'encontre du droit européen, d'interrompre immédiatement les conditions d'accueil sans que les personnes aient eu la possibilité de présenter des observations ${ }^{16}$ ».

11 L'explication de cette absence ne peut être qu'hypothétique. Elle réside peut-être dans le lien entre l'orientation active des demandeurs d'asile et le retrait des conditions matérielles d'accueil. L'étude d'impact a indiqué que l'objectif de l'article 9 était d' « améliorer le pilotage du dispositif national d'accueil » notamment en « [simplifiant] les modalités de retrait ou de limitation des conditions matérielles d'accueil ${ }^{17}{ }^{7}$. Le 
projet de loi et les débats qui l'ont entouré furent indifférents à la situation particulière des demandeurs d'asile et ne considèrent la question du retrait que sous l'angle de l'efficacité du dispositif et de la réalisation des objectifs de politiques publiques. Ce lien a conditionné le point de vue sur la procédure de retrait. Elle ne fut envisagée qu'en raison des risques de ralentissement qu'elle faisait peser sur l'action administrative. La suppression de procédure préalable contradictoire permettrait de rendre efficace l'orientation active des demandeurs d'asile. En revanche, du point de vue de la gestion administrative d'une politique publique, l'exigence de prise en compte de la situation particulière du demandeur d'asile est un problème secondaire pouvant être ignoré.

\section{B. La qualification du retrait des conditions matérielles d'accueil}

13 Quels que soient les causes de cette erreur, la section de l'intérieur du Conseil d'État n'y échappa pas. Dans son avis sur l'avant-projet de loi, elle n'a émis qu'une critique à l'encontre de l'article 9. L'avant-projet de loi prévoyait que l'ensemble des procédures de retrait aurait lieu sans procédure contradictoire préalable. Or, dans certains cas, les retraits du bénéfice des conditions matérielles d'accueil d'un demandeur d'asile peuvent être des sanctions administratives. Une telle qualification implique que ces retraits soient précédés d'une procédure contradictoire préalable afin de garantir le principe constitutionnel du respect des droits de la défense ${ }^{18}$. Le Conseil d'État a alors opéré une distinction. Les retraits fondés sur l'article L. 744-8 c'est-à-dire résultant du fait qu'un demandeur d'asile a fourni des informations mensongères à l'administration, a eu un comportement violent ou manquant aux règles de son lieu d'hébergement ou a présenté plusieurs demandes d'asile sous plusieurs identités différentes, sont des sanctions administratives. En revanche, «l'abandon du logement ou la cessation de résidence dans la région désignée ne [doivent pas être] regardés comme une sanction, nécessairement précédée d'une procédure contradictoire, mais comme la cessation de plein droit d'un avantage, si les règles d'attribution des conditions matérielles d'accueil sont rédigées de manière à faire apparaître qu'elles résultent d'un choix du demandeur d'asile, dûment informé des conséquences de celui-ci ${ }^{19} »$. De tels retraits ont été alors réunis à l'article L. 744-7.

14 L'analyse de la section de l'intérieur du Conseil d'État découle du critère qui caractérise une sanction administrative: avoir pour motif le fait personnel d'un individu ${ }^{20}$. Les retraits fondés sur l'article L. 744-7 ne résulteraient pas d'un «comportement particulier ${ }^{21} » d u$ demandeur d'asile. Certes, ces retraits sont déclenchés par le comportement d'un individu - quitter son logement par exemple mais l'administration se bornerait à constater «que les conditions d'attribution d'un droit [- le bénéfice des conditions matérielles d'accueil - ] initialement prévues [ conserver son logement et se rendre aux rendez-vous prévus par la Préfecture -] ne sont plus remplies ${ }^{22}$ ». Elle ne ferait que tirer "les conséquences juridiques d'une situation donnée, à savoir que l'intéressé ne remplit pas ou plus les conditions fixées par la loi $^{23}$ ». Il ne s'agirait pas d'une sanction mais du constat de la caducité d'un acte ${ }^{24}$. Les exigences issues du principe constitutionnel de respect du droit de la défense ne pèsent dès lors pas sur le législateur. Par conséquent, le retrait peut être immédiat, sans procédure préalable contradictoire et sans appréciation au cas par cas de la situation du demandeur d'asile. 
15 L'analyse de la section de l'intérieur du Conseil d'État appelle deux remarques. D'abord, le texte de la directive est ambigu. Le paragraphe 4 de l'article 20 dispose que «les États membres peuvent déterminer les sanctions applicables en cas de manquement grave au règlement des centres d'hébergement ainsi que de comportement particulièrement violent" tandis que le paragraphe 5 oppose «les décisions portant limitation ou retrait du bénéfice des conditions matérielles d'accueil ou les sanctions visées aux paragraphes 1, 2, 3 et 4 ». La directive paraît ainsi tracer une distinction entre deux types de décisions. Mais d'une part, ainsi que le relève l'avocat général Manuel Campos Sànchez-Bordona dans ses conclusions sur l'arrêt Haqbin, il ne s'agit que d'une interprétation possible de la directive, la Cour de justice n'ayant jamais eu à trancher ce problème ${ }^{25}$. D'autre part, si cette interprétation devait être retenue, elle ne concernerait que les retraits en raison d'un "manquement grave aux règlements des centres d'hébergement" ou d'un "comportement particulièrement violent ». Or, l'article L. 744-8 inclut d'autres cas de figure tel que le retrait motivé par la multiplication de demandes d'asiles en utilisant plusieurs identités. La section de l'intérieur du Conseil d'État ne pourrait donc justifier son découpage en s'appuyant sur la directive. Ensuite, dans les travaux préparatoires ayant abouti à la refonte de la directive Accueil, le retrait du bénéfice des conditions matérielles d'accueil a toujours été considéré comme une sanction visant à sanctionner les abus ${ }^{26}$. Dans les rapports d'organisations non-gouvernementales ${ }^{27}$ ou du Haut Commissariat des Nations-Unies pour les réfugiés ${ }^{28}$, les retraits sont aussi systématiquement qualifiés ainsi. De même, dans les autres États membres, les retraits sont conçus comme des sanctions ${ }^{29}$. Le refus de qualifier de sanction administrative les retraits fondés sur l'article L. 744-7 n'était ainsi guère évident.

16 L'avis de la section de l'intérieur du Conseil d'État résulte en partie du contexte de la loi du 10 septembre 2018. Les évolutions de la procédure de retrait des conditions matérielles d'accueil y furent conçues afin de permettre l'efficacité de l'orientation active des demandeurs d'asile. Cette efficacité amena le Gouvernement, le Parlement ainsi que le Conseil d'état à ne considérer que la suppression de la procédure préalable contradictoire. Cette suppression fut examinée au travers du principe constitutionnel du respect des droits de la défense et de l'interdiction de sanctionner un individu sans l'avoir autorisé à présenter ses vues au préalable. En concevant les retraits fondés sur l'article L. 744-7 uniquement comme le résultat du constat objectif de l'absence de réalisation des conditions d'obtention des conditions matérielles d'accueil, le Conseil d'État a estimé qu'il ne s'agissait pas de sanctions administratives. Dès lors, les retraits pouvaient être immédiats et de pleins droits. Mais ce raisonnement ne résista pas à la confrontation de la procédure aux exigences du droit de l'Union.

\section{2./ L'inconventionalité des retraits immédiats et de plein droit}

17 Dès son entrée en vigueur, l'article L. 744-7 fut employé à une fin distincte de son objet initial - permettre la répartition des demandeurs d'asile. Il fut utilisé pour fonder des refus de rétablir le bénéfice des conditions matérielles d'accueil au profit d'un demandeur d'asile dont les conditions d'accueil ont été suspendues pendant la procédure Dublin ${ }^{30}$. Ces refus créèrent un contentieux ayant confronté le Conseil d'État aux difficultés soulevées par cette loi et par son décret d'application au regard du droit 
de l'Union européenne. En effet, dans la directive Accueil et la jurisprudence de la Cour de justice, des obligations de procéder à une appréciation au cas par cas de la situation des demandeurs d'asile afin de prendre en compte leur vulnérabilité (A) et de permettre aux demandeurs d'asile de présenter ses vues préalablement à tout retrait (B) ont été reconnues. Ignorés lors de l'examen du projet de loi, ces éléments conduisirent au renversement de la solution retenue par la section de l'intérieur.

\section{A. L'exigence d'appréciation au cas par cas de la situation des demandeurs d'asile}

18 Le paragraphe 5 de l'article 20 de la directive Accueil est explicite. Toutes les décisions de retrait doivent être "prises au cas par cas» et être "fondées sur la situation particulière de la personne concernée ». Des retraits indépendants d'un comportement particulier du demandeur d'asile et ne prenant pas en compte son éventuelle vulnérabilité, sont incompatibles avec cette exigence. L'examen des travaux préparatoires de la directive révèle l'ampleur de cette opposition.

19 L'un des objectifs fondamentaux de la refonte du régime d'asile européen commun fut la meilleure prise en compte des vulnérabilités ${ }^{31}$. Cet objectif guida les discussions relatives à la réforme du retrait des bénéfices des conditions matérielles d'accueil. Dans l'une de ses analyses d'impact, la Commission a considéré qu'à l'instar de la directive de 2003, la proposition de directive devait autoriser les États membres à réduire ou retirer le bénéfice des conditions matérielles d'accueil. Néanmoins, elle a constaté que lors de la mise en œuvre de cette faculté, les États membres ont été confrontés au risque de provoquer des traitements inhumains ou dégradants. En particulier, la House of Lords du Royaume-Uni a censuré la politique britannique consistant à retirer systématiquement le bénéfice des conditions matérielles d'accueil aux demandeurs d'asile échouant à prouver qu'il avait introduit une demande d'asile dans les trois jours suivant leur arrivée sur le territoire britannique. La House of Lords a exigé le recours à une évaluation de tous les faits et circonstances permettant d'établir si un demandeur d'asile ferait face à un risque grave et imminent en raison de la privation du bénéfice des conditions matérielles d'accueil ${ }^{32}$. Dès lors, la Commission a estimé nécessaire d'encadrer la marge d'appréciation des États membres. Les décisions de retrait ne devaient pas conduire à laisser les demandeurs d'asile sans ressources et devaient protéger les demandeurs d'asile ayant des besoins particuliers ${ }^{33}$. Deux options furent alors considérées par la Commission: interdire tout retrait et diminution des conditions matérielles ou maintenir cette possibilité mais en exigeant que les retraits résultent d'une évaluation circonstanciée et motivée. Par conséquent, les retraits tels que ceux mis en place par l'article L. 744-7 furent exclus. La Commission a relevé qu'il en allait notamment ainsi car cela permettait de garantir les droits fondamentaux des demandeurs d'asile et en particulier, leur droit à la vie et le respect de leur dignité humaine ${ }^{34}$.

20 L'exigence d'appréciation concrète au cas par cas lors des retraits est ainsi inscrite dans la lettre et dans l'esprit de la directive. Le Conseil d'état y fut indirectement confronté lors de son examen des refus de rétablir le bénéfice des conditions matérielles d'accueil après des décisions de retraits adoptées sur le fondement de l'article L. 744-8 avant sa modification par la loi du 10 septembre 2018. Dans sa décision de principe, il a insisté sur la nécessité d'une appréciation au cas par cas prenant en 
compte la vulnérabilité des demandeurs d'asile dans les décisions de retrait et lors de l'examen des demandes de rétablissement ${ }^{35}$. L'arrêt du 31 juillet 2019 n'a fait qu'appliquer cette exigence aux modalités de retrait issues de la loi du 10 septembre 2018. En affirmant que «le retrait des conditions matérielles d'accueil] ne [pouvait] intervenir qu'après examen de la situation particulière de la personne", le Conseil d'État a transposé l'obligation explicitement posée par l'article 20, paragraphe 5, de la directive. La décision de retirer le bénéfice des conditions matérielles d'accueil ne pouvait pas se réduire au constat la non-réalisation d'une condition. Elle ne pouvait être que le produit d'une appréciation au cas par cas laissant une marge d'appréciation à l'administration. De cette obligation explicite, le Conseil d'État a déduit une seconde obligation. Puisque les retraits étaient le produit d'une appréciation au cas par cas en vue de prendre compte notamment la vulnérabilité des demandeurs d'asile, ces derniers devaient pouvoir demander le rétablissement des conditions matérielles d'accueil lorsque leur situation avait évolué. Cette exigence est absente du texte et des travaux préparatoires de la directive mais elle résulte de ce que le Conseil d'État a nommé « les objectifs de la directive ${ }^{36}$ ».

21 Lors de son contrôle a posteriori, le Conseil d'État a ainsi écarté la solution retenue par la section de l'intérieur qui avait déclarée la loi conforme aux exigences conventionnelles. Mais l'opposition entre les deux solutions va plus loin. Dans sa décision du 31 juillet 2019, le Conseil d'État ne s'est pas limité à examiner des éléments ignorés par la section de l'intérieur. Il a contredit frontalement cette dernière en réintégrant une obligation de procédure contradictoire préalable aux retraits.

\section{B. L'obligation d'une procédure contradictoire préalable}

22 En effet, après avoir constaté l'incompatibilité des articles L. 744-7 et L. 744-8 avec la directive, le Conseil d'État en a exposé les conséquences. En particulier, dans l'attente d'une nouvelle loi, l'office français de l'immigration et de l'intégration peut «après avoir mis, sauf impossibilité, l'intéressé en mesure de présenter ses observations, [décider] de suspendre le bénéfice de ces conditions lorsque le demandeur a quitté le lieu d'hébergement proposé ou la région d'orientation ou n'a pas respecté les exigences des autorités chargées de l'asile, notamment de se rendre aux entretiens, de se présenter aux autorités et de fournir les informations utiles afin de faciliter l'instruction des demandes $^{37}$ ». Alors que la section de l'intérieur Conseil d'État avait réécrit l'article 9 de l'avant-projet de loi afin de permettre que les retraits fondés sur l'article L. 744-7 puissent avoir lieu en l'absence de procédure préalable contradictoire, le Conseil d'État a exigé au contraire que ces retraits fussent précédés d'une telle procédure.

23 Une question demeure. Cette exigence ne peut être déduite du principe constitutionnel de respect des droits de la défense ou de l'article 6 de la Convention européenne des droits de l'homme dont les obligations ne s'appliquent qu'aux procédures d'adoption d'une sanction administrative. La directive est silencieuse sur ce point. L'exigence d'une appréciation au cas par cas n'est pas nécessairement liée à celle d'une procédure préalable contradictoire. Par ailleurs, l'article 41, paragraphe 2, de la Charte des droits fondamentaux de l'Union européenne reconnaît «le droit de toute personne d'être entendue avant qu'une mesure individuelle qui l'affecterait défavorablement ne soit prise à son encontre ». Cependant, le paragraphe 1 précise que les droits garantis à l'article 41, ne s'applique qu'aux institutions et organes de l'Union. Bien qu'ils ne 
fussent pas visés par la décision du Conseil d'État, la seule source possible de cette exigence est alors les principes généraux du droit de l'Union. Le droit d'être entendu avant toute décision défavorable est un aspect du respect des droits de la défense garantie en tant que principe général du droit ${ }^{38}$. Par conséquent, « cette obligation pèse sur les administrations des États membres lorsqu'elles prennent des décisions entrant dans le champ d'application du droit communautaire, alors même que la législation communautaire applicable ne prévoit pas expressément une telle formalité39 ». Malgré le silence de la directive, l'État doit donc être considéré comme tenu de permettre aux demandeurs d'asile de présenter leurs vues avant toute décision de retraits du bénéfice des conditions matérielles d'accueil. Appliquant les principes généraux du droit de l'Union, le Conseil d'État a ainsi renversé intégralement l'avis de la section de l'intérieur.

\section{NOTES}

1. CE, 17 avril 2019, req. $\mathrm{n}^{\circ} 428314$, publié au Recueil Lebon ; CE, ord., 19 avril 2019, req. $\mathrm{n}^{\circ} 428038$, inédit au Recueil Lebon ; CE, ord., 26 avril 2019, req. n 429945, inédit au Recueil Lebon ; CE, ord., 9 juillet 2019, req. $n^{\circ} 431937$, inédit au Recueil Lebon ; CE, ord.,9 juillet 2019, req. $n^{\circ} 431695$, inédit au Recueil Lebon.

2. CE, 31 juillet 2019, Cimade et al., req. $n^{\circ} 428530$, publié au Recueil Lebon.

3. Directive 2013/33/UE du Parlement européen et du Conseil du 26 juin 2013 établissant des normes pour l'accueil des personnes demandant la protection internationale.

4. Étude d'impact du projet de loi pour une immigration maîtrisée et un droit d'asile effectif, 20 février 2018, NOR : INTX1801788L/Bleue-1, p.64.

5. Ainsi, l' article L.744-8 débute en opposant les cas mentionnés à l'article L. 744-7, «il est immédiatement mis fin de plein droit au bénéfice des conditions matérielles d'accueil » et ceux de l'article L.744-8.

6. Sur le caractère vague de l'expression « de plein droit », voir notamment Christian ATIAS, « De plein droit », 2013, p. 2183.

7. CE, 31 juillet 2019, Cimade et al., précité, cons. 11. Un point doit être précisé. Le Conseil d'État a visé indifféremment les article L. 744-7 et L. 744-8. Mais seul les retraits sur le fondement de l'article L. 744-7 étaient en cause. En effet, l'article L. 744-8 n'a été visé qu'en raison du fait que sa première phrase précisait le régime de l'article L. 744-7. Afin d'éviter toute confusion, nous nous permettrons pour la suite de ce développement de ne viser que l'article L. 744-7.

8. Il disposait que "l'offre de prise en charge faite au demandeur d'asile en application de l'article L. 744-1 fait mention de la possibilité pour le demandeur d'asile de se voir refuser, retirer 
le bénéfice des conditions matérielles d'accueil ou qu'il y soit mis fin dans les conditions prévues par la présente sous-section ».

9. CE, 31 juillet 2019, Cimade et al., précité, cons. 15.

10. CE, 15 février 2018, Avis sur un projet de loi une immigration maîtrisée et un droit d'asile effectif, $\mathrm{n}^{\circ} 394206$, pt. 29. Le Conseil constitutionnel fut aussi saisi mais les auteurs de la saisine ne soulèvent aucun moyen ayant trait aux retraits du bénéfice des conditions matérielles d'accueil (Cons. Const., 6 septembre 2018, décision n²018-770 DC, Loi une immigration maitrisée, un droit d'asile effectif et une intégration réussie).

11. Intervention de M. Guillaume Gontard lors de la séance publique du 21 juin 2018 au Sénat.

12. «Le d) du $5^{\circ}$ prévoit une procédure contradictoire spécifique dans les cas de retrait, de suspension ou de refus des conditions matérielles d'accueil ».

13. Étude d'impact du projet de loi pour une immigration maîtrisée et un droit d'asile effectif, 20 février 2018, NOR : INTX1801788L/Bleue-1, p.62.

14. Avis du Défenseur des droits, 15 mars 2018, n¹8-09, p.16.

15. Une exception: le 4 juin 2018, la Commission des lois a adopté l'amendement COM-247 proposé par le Sénateur François-Noël Buffet visant à créer une compétence liée aussi pour les cas prévus par l'article L. 744-8. L'Assemblée nationale revint ensuite sur cette solution.

16. Rapport de la Cimade, 21 février 2018, Décryptage du projet de loi Asile et immigration, p.4. Les analyses du GISTI ou d'Amnesty International sont elles aussi silencieuses sur ce point.

17. Étude d'impact du projet de loi pour une immigration maîtrisée et un droit d'asile effectif, 20 février 2018, NOR : INTX1801788L/Bleue-1, p.61.

18. Cons. Const., 28 décembre 1990, décision $n^{\circ}$ 90-285 DC, Loi de finances pour 1991, cons. 55-56. L'histoire de la reconnaissance de ce principe est connue. Dès 1905, une loi a imposé à l'administration l'obligation de transmettre son dossier à un fonctionnaire avant toute sanction disciplinaire. En effet, en matière de sanctions disciplinaires. En 1930 dans une décision Ribeyrolles (CE, Sect., 17 janvier 1930, Ribeyrolles, publié au Recueil Lebon) et surtout dans une décision Dame veuve Trompier Gravier (CE, Sect., 5 mai 1944, Dame veuve Trompier-Gravier, req. n69751, publié au Recueil Lebon), le Conseil d'État étendit cette exigence au-delà des seuls agents publics. Dans une décision Aramu, il reconnut l'existence d'un principe général du droit selon lequel « une sanction ne peut [...] être prononcée légalement sans que l'intéressé ait été mis en mesure de présenter utilement sa défense » (CE, Ass., 26 octobre 1945, Aramu et a., req. $\mathrm{n}^{\circ} 77726$, publié au Recueil Lebon). La portée de l'exigence de procédure contradictoire préalable a été étendue à l'ensemble des décisions administratives individuelles, défavorables et prises en considération de la personne (CE, Ass., 31 octobre 1952, Ligue pour la protection des mères abandonnées, publié au Recueil Lebon; CE, Sect., 8 janvier 1960, Ministère de l'intérieur contre Rohmer, publié au Receuil Lebon ; CE, Sect., 9 mai 1980, req. n¹0404, Société des établissements Cruse fils et frère, publié au Recueil Lebon). Mais ce principe n'a qu'une valeur législative lorsque n'est pas en cause une sanction (Cons. Const., 27 novembre 2011, décision n²001-451 DC, Loi portant amélioration de la couverture des non salariés agricoles contre les accidents du travail et les maladies professionnelles, cons. 40). Voir Pascale IDOUX, La contradiction en droit administratif français, Montpellier : Université Montpellier I, 2005, p. 237 et suiv.

19. CE, 15 février 2018, Avis sur un projet de loi une immigration maîtrisée et un droit d'asile effectif, n³94206, pt. 32.

20. Jean-Marie AUBY, «La procédure administrative non contentieuse », D., 1956, p. 27-32 ; Claude-Albert COLLIARD, «La sanction administrative ", Annales de la faculté d'Aix-en-Provence, $\mathrm{n}^{\circ}$ 36, 1943, p. 5 ; Mireille Delmas-MARTy et Catherine teItGen-Colly, Punir sans juger: de la répression administrative au droit administratif pénal, Paris : Economica, 1992, p. 44 ; Franck MODERNE, Sanctions administratives et justice constitutionnelle - Contribution à l'étude du jus puniendi de l'État dans les démocraties contemporaines, Paris: Economica, 1999, p. 79; Jacques MOURGEON, La répression administrative, Paris : Librairie générale de droit et de jurisprudence, 1967, p. 129 et suiv. ; Jean- 
Pierre MUNCH, La sanction administrative, Thèse de doctorat, Université de Paris, Paris, 1947, p. 129 ; René ODENT, «Les droits de la défense ", Études et documents du Conseil d'État, 1953, p. 62 ; Étienne PICARD, La notion de police administrative, Paris : L.G.D.J., 1984 (vol. 1/2), p. 357 ; Jacques PUISOYE, « La jurisprudence sur le respect des droits de la défense devant l'administration ", AJDA, $\mathrm{n}^{\circ} 1,1962$, p. 79-85.

21. Exposé des motifs du projet de loi.

22. Étude d'impact du projet de loi pour une immigration maîtrisée et un droit d'asile effectif, 20 février 2018, NOR : INTX1801788L/Bleue-1, p.65.

23. Ibid.

24. Un parallèle peut être trace avec les décisions du CSA retirant une autorisation d'émettre sur une fréquence radioélectrique (CE, Sect., 22 mars 1996, N.R.J. S.A., req. n¹31861, publié au Recueil Lebon). En ce sens, voir Franck MODERNE, « Le pouvoir de sanction administrative au confluent du droit interne et des droits européens », RFDA, n 1, 1997, p. 1.

25. Conclusions de l'avocat général Manuel Campos Sànchez-Bordona dans l'affaire Zubair Haqbin contre Federaal agentschap voor de opvang van asielzoekers, C-233/18, pts. 42-59. Il s'agit de l'unique affaire dans laquelle cette question fut soulevée à notre connaissance. Au moment où nous écrivons, la CJUE n'a pas encore rendu sa décision.

26. Voir notamment Commission Staff Working accompanying the Proposal for a Directive of the European Parliament and of the Council layind down minimum standards for the reception of asylum seekers, 3 décembre 2008, SEC (2008) 2944, p.1 et 15.

27. Voir ainsi ECRE Comments on the Commission Proposal for a Dublin IV Regulation COM(2016) 270, Bruxelles : European Council on Refugees and Exilee, 2016, p. 5. URL : 23/10/2019; Withdrawal of Reception Conditions of Asylum Seeker. An Appropriate, Effective or Legal Sanction?, Bruxelles : AIDA, 2019, p. $2 . \quad$ URL: https://www.asylumineurope.org/sites/default/files/ aida_brief_withdrawalconditions.pdf. Consulté le 23 novembre 2019.

28. UNHCR Annotated Comments to Directive 2013/33/EU of the European Parliament and Council of 26 June 2013 laying down standards for the reception of applicants for international protection (recast), Genève : UNHCR, 2015, p. 48. URL : https://www.refworld.org/docid/5541d4f24.html. Consulté le 21 octobre 2019.

29. Voir sur ce point, les analyses États par États effectués sur le site de l'Asylum Information Database (https://www.asylumineurope.org/about-aida).

30. Par exemple, CE, ord., 19 avril 2019, req. $n^{\circ} 428038$, précité ; CE, ord., 26 avril 2019, req. $n^{\circ}$ 429945, précité ; CE, ord., 9 juillet 2019, req. n 431937, précité ; CE, ord.,9 juillet 2019, req. n431695, précité.

31. Par exemple, parmi les 4 objectifs stratégiques de la refonte de la directive Accueil, la Commission indiqua qu'il était nécessaire de " garantir un niveau de vie digne, en prévoyant des mesures nationales de détermination des besoins particuliers des personnes vulnérables, telles que les mineurs et les victimes d'actes de torture, et en assurant aux demandeurs d'asile un niveau approprié d'aide matérielle " (Commission, communiqué de presse, 1 juin 2011, IP/ 11/665).

32. House of Lords, 3 novembre 2005, R(Q) v Secretary of State for the Home Department [2004] QB 36, pt. 8

33. Commission Staff Working accompanying the Proposal for a Directive of the European Parliament and of the Council layind down minimum standards for the reception of asylum seekers, 3 décembre 2008, SEC (2008) 2944, p.15. L'expression "personne vulnérable" fut introduite plus tardivement dans le processus législatif.

34. Commission Staff Working accompanying the Proposal for a Directive of the European Parliament and of the Council layind down minimum standards for the reception of asylum seekers, 3 décembre 2008, SEC (2008) 2944, p.45.

35. CE, 17 avril 2019, req. ${ }^{\circ} 428314$, précité, cons. 7. 
36. Ibid., cons. 10 .

37. Ibid., cons. 18.

38. CJCE, 24 octobre 1996, Commission des Communautés européennes contre Lisrestal - Organização Gestão de Restaurantes Colectivos $L d^{\underline{a}}$, Gabinete Técnico de Informática Ld $d^{a}$ (GTI), Lisnico - Serviço Marítimo Internacional $L d^{\underline{a}}$, Rebocalis - Rebocagem e Assistência Marítima $L d^{a}$ et Gaslimpo - Sociedade de Desgasificação de Navios SA, aff. C-32/95 P, Rec. p.I-5373, pt.21. Il y a lieu de relever que ce raisonnement est maintenu malgré l'adoption de la Charte. Voir CJUE,, gde. ch., 22 octobre 2013, Jiř́ Sabou contre Finanční ředitelství pro hlavní město Prahu, aff. C-276/12, publié au Recueil numérique, pt. 38. Voir aussi CJUE, 5 novembre 2014, Sophie Mukarubega contre Préfet de police et Préfet de la Seine-Saint-Denis, aff. C-166/13, publié au Recueil numérique, pt.45 et CJUE, 17 décembre 2017, WebMindLicenses Kft. contre Nemzeti Adó- és Vámhivatal Kiemelt Adoés Vám Főigazgatóság, aff. C-419/14, publié au Recueil numérique, pt. 84. Une telle décision démontre l'utilité du maintien des principes généraux du droit de l'Union en vue de garantir les droits fondamentaux, la Charte comportement un certain nombre d'angles morts dans la protection des droits. Sur ce point, voir Françoise TULKENS, "Article 41 », in Fabrice PICOD et Sébastien VAN DROOGHENBROECK (dirs.), Charte des droits fondamentaux de l'Union européenne - Commentaire article par article, Bruxelles : Bruylant, 2017, p. 879 ; Claire VIAL, «Cour de justice, 5e ch., 5 novembre 2014, Mukarubega, aff. C-166/13, ECLI:EU:C:2014:2336», in Fabrice PICOD (dir.), Jurisprudence de la CJUE 2014, Bruxelles : Bruylant, 2014, p. 121-123.

39. CJCE, 18 décembre 2008, Sopropé - Organizações de Calçado Lda contre Fazenda Pública, aff. C-349/07, Rec. p.I-10369, pt.38.

\section{ABSTRACTS}

Par un jugement en date du 31 juillet 2019, le Conseil d'Etat juge que l'inconventionnalité partielle de la loi Asile et immigration du 10 septembre 2018 emporte l'illégalité de deux dispositions du décret $n^{\circ}$ 2018-1359 du 28 décembre 2018 relatif aux conditions matérielles d'accueil des demandeurs d'asile. Le Conseil d'État indique que, s'il est possible dans des cas exceptionnels et dûment justifiés de retirer les conditions matérielles d'accueil à un demandeur d'asile, ce retrait ne peut intervenir qu'après examen de la situation particulière de la personne et être motivé. D'autre part l'intéressé doit pouvoir solliciter le rétablissement des conditions matérielles d'accueil lorsque le retrait a été fondé sur l'abandon du lieu de résidence sans information ou autorisation de l'autorité compétente, sur la méconnaissance de l'obligation de se présenter aux autorités ou de se rendre aux rendez-vous qu'elle fixe ou sur l'absence de réponse aux demandes d'information.

\section{AUTHOR}

PIERRE AURIEL

Docteur en droit public, Université Paris 2 Panthéon-Assas 